\title{
ON ROGUE WAVE IN THE KUNDU-DNLS EQUATION
}

\author{
SHIBAO SHAN $\dagger$, CHUANZHONG LI $\dagger$, JINGSONG HE †‡ \\ $\dagger$ Department of Mathematics, Ningbo University, Ningbo, 315211, China
}

\begin{abstract}
In this paper, the determinant representation of the n-fold Darboux transformation (DT) of the Kundu-DNLS equation is given. Based on our analysis, the soliton solutions, positon solutions and breather solutions of the Kundu-DNLS equation are given explicitly. Further, we also construct the rogue wave solutions which are given by using the Taylor expansion of the breather solution. Particularly, these rogue wave solutions possess several free parameters. With the help of these parameters, these rogue waves constitute several patterns, such as fundamental pattern, triangular pattern, circular pattern.
\end{abstract}

PACS numbers: 42.65.Tg, 42.65.Sf, 05.45.Yv, 02.30.Ik.

Keywords: Kundu-DNLS equation, Darboux transformation, soliton solutions, breather solution, positon solution, rogue waves.

\section{INTRODUCTION}

It is well known that the derivative nonlinear Schrödinger (DNLS) equation

$$
i q_{t}+q_{x x}-i \alpha\left(|q|^{2} q\right)_{x}=0
$$

is one of the most important integrable systems in many branches of physics and applied mathematics, particularly in optics, water wave and so on [1,2]. In the eq.(1.1), $q$ represents complex field envelope and the subscripts imply the partial derivative with regard to $x$ or $t$. Considering the significance of the higher order nonlinearities in physical system, the DNLS equation yields an integrable higher nonlinear equation, i.e. Kundu-DNLS equation [3, 4]

$$
i Q_{t}+Q_{x x}+i \alpha\left(Q^{2} Q^{*}\right)_{x}-\left(\theta_{t}+\theta_{x}^{2}-i \theta_{x x}\right) Q+\theta_{x}\left(2 i Q_{x}-\alpha Q^{2} Q^{*}\right)=0
$$

by means of a nonlinear transformation of the field $q \rightarrow Q=q e^{-i \theta}$ with arbitrary gauge function $\theta$. Here $Q^{*}$ denotes the complex conjugate of $\mathrm{Q}$, and $\alpha$ is a real parameter. For example, setting $\theta=\delta \int Q\left(x^{\prime}\right)^{2} d x^{\prime}$, Kundu-DNLS equation implies Eckhaus-Kundu (EK) equation [3]. There are several method to solve the integrable equations, for instance, Hirota method [5], inverse scattering transformation [6], bilinear method [7], Darboux transformation [8]. Among these methods, the Darboux transformation has an old history, which originates from the paper of Darboux in 1882 about the study of the Sturn-Liouville equation, and is a efficient method to construct the solutions for the integrable systems. To a given equation, there are also many approaches to find the corresponding Darboux transformation, such as the gauge transformation method [8 11], the operator factorization method [12]. In this paper, in terms of the Darboux transformation, we find that this equation can also be used to describe many intriguing physical phenomena, and possessing the soliton solutions, breather solution, positon solution, the rogue waves.

Before considering the Darboux transformation, let us briefly discuss the importance of rogue waves in mathematical field and physical field. Rogue waves, appearing in oceans "appears from

$\ddagger$ Corresponding author. 
nowhere and disappears without a trace [13]", which cause a large number of disasters for people. Consequently, it appeals to much more and more attention for many scientists and has been studied extensively in other fields such as optics [14 16], Bose-Einstein condensates [17], femtosecond pulse [18,19] propagation due to the modulation instability [20]. The higher order rogue waves of the nonlinear Schrödinger (NLS) equation have interesting patterns [21 25. Recently, we have given the rogue waves of the DNLS and the coupled system of Hirota and Maxwell-Bloch equations [2, 26], Inspired by the importance of these recent interesting developments about the analysis of rogue waves of the NLS-type equations, we shall also construct the rogue wave solutions of the Kundu-DNLS equation with the help of the Darboux transformation.

The paper is organized as follows. In Section 2, the Darboux transformation and the Lax pair of the Kundu-DNLS equation will be introduced firstly. We also derived the one-fold Darboux transformation of the Kundu-DNLS equation. And the determinant-formed generalization of one-fold Darboux transformation to 2-fold Darboux transformation of the Kundu-DNLS equation will be given. In Section 3, by making use of Darboux transformation, one soliton, two soliton, positon solution, are derived. With the help of these formulas, breather solution, and rogue waves are derived in Section 4. In Section 5, conclusions are given.

\section{Darboux transformation AND LAX PAiR}

The Darboux transformation is a powerful method used to generate the soliton solutions for integrable equations. Inspired by classical Darboux transformation for the DNLS equation [27 32, we consider the coupled Kundu-DNLS equation,

$$
\begin{aligned}
& i Q_{t}+Q_{x x}-i \alpha\left(Q^{2} R\right)_{x}-\left(\theta_{t}+\theta_{x}^{2}-i \theta_{x x}\right) Q+\theta_{x}\left(2 i Q_{x}+\alpha Q^{2} R\right)=0, \\
& i R_{t}-R_{x x}-i \alpha\left(R^{2} Q\right)_{x}+\left(\theta_{t}+\theta_{x}^{2}+i \theta_{x x}\right) R+\theta_{x}\left(2 i R_{x}-\alpha R^{2} Q\right)=0,
\end{aligned}
$$

where $\theta$ is a arbitrary gauge function. This form of the equation is very extensive, which is reduced to the eq.(1.2) for $R=-Q^{*}$ with the sign of the nonlinear term changed. The Kundu-DNLS equation can be obtained if $\alpha$ is a real parameter.

We first present a general framework for deriving the required conservation rule for the DNLS equation. We start with the linear set of Lax equations:

$$
\Phi_{x}=U \Phi, \Phi_{t}=V \Phi
$$

where $U$ and $V$ depend on the complex constant eigenvalue parameter $\lambda$

$$
\begin{aligned}
U & =i \frac{\lambda^{2}}{4}\left(\begin{array}{cc}
1 & 0 \\
0 & -1
\end{array}\right)+\frac{i}{2} \lambda \sqrt{\alpha}\left(\begin{array}{cc}
0 & R e^{-i \theta} \\
Q e^{i \theta} & 0
\end{array}\right), \\
V & =i\left(\frac{\lambda^{4}}{8}-\frac{\alpha}{4} \lambda^{2} Q R\right)\left(\begin{array}{cc}
1 & 0 \\
0 & -1
\end{array}\right)+i\left(\begin{array}{cc}
0 & G^{*} \\
G & 0
\end{array}\right),
\end{aligned}
$$

with $G=\frac{\lambda}{4} \sqrt{\alpha}\left(-\lambda^{2} Q e^{i \theta}+2 i\left(Q_{x} e^{i \theta}+i Q e^{i \theta} \theta_{x}\right)+2 \alpha Q^{2} Q^{*} e^{i \theta}\right)$, where $\lambda$ is the eigenvalue, $\Phi$ is the eigenfunction corresponding to $\lambda$.

Next, we will give the detailed proof for the one-fold Darboux transformation of Kundu-DNLS equation. Equations(2.1) and (2.2) are equivalent to the integrability condition $U_{t}-V_{x}+[U, V]=$ 0 of (2.3). we would like to introduce a simple gauge transformation of the spectral problem (2.3) with the following form

$$
\psi^{[1]}=T \psi
$$


It can transform linear equation (2.3) into a new one possessing the same matrix form, namely,

$$
\begin{aligned}
& \psi_{x}^{[1]}=U^{[1]} \psi^{[1]}, \quad U^{[1]}=\left(T_{x}+T U\right) T^{-1}, \\
& \psi_{t}^{[1]}=V^{[1]} \psi^{[1]}, \quad V^{[1]}=\left(T_{t}+T V\right) T^{-1},
\end{aligned}
$$

where $U^{[1]}, V^{[1]}$ have the similar forms as $U, V$. By cross differentiating (2.6) and (2.7), we obtain

$$
U_{t}^{[1]}-V_{x}^{[1]}+\left[U^{[1]}, V^{[1]}\right]=T\left(U_{t}-V_{x}+[U, V]\right) T^{-1} .
$$

2.1. One-fold Darboux transformation. In general, considering the universality of Darboux transformation, according to the Kundu-DNLS equation (2.1) and (2.2), we can start from

$$
T=T(\lambda)=\left(\begin{array}{ll}
a_{2} & b_{2} \\
c_{2} & d_{2}
\end{array}\right) \lambda^{2}+\left(\begin{array}{ll}
a_{1} & b_{1} \\
c_{1} & d_{1}
\end{array}\right) \lambda+\left(\begin{array}{ll}
a_{0} & b_{0} \\
c_{0} & d_{0}
\end{array}\right),
$$

where $a_{0}, b_{0}, c_{0}, d_{0}, a_{1}, b_{1}, c_{1}, d_{1}, a_{2}, b_{2}, c_{2}, d_{2}$ are functions of $x, t$. From

$$
T_{x}+T U=U^{[1]} T,
$$

comparing the coefficients of $\lambda^{j}, j=4,3,2,1,0$, it yields

$$
\begin{aligned}
& \lambda^{4}: b_{2}=0, c_{2}=0, \\
& \lambda^{3}:-d_{2} \sqrt{\alpha} Q+c_{1} e^{i \theta}+a_{2} \sqrt{\alpha} Q^{[1]}=0, d_{2} \sqrt{\alpha} R^{[1]}-b_{1} e^{-i \theta}-a_{2} \sqrt{\alpha} R=0, \\
& \lambda^{2}:-d_{1} \sqrt{\alpha} Q e^{i \theta}+c_{0}+a_{1} \sqrt{\alpha} Q^{[1]} e^{i \theta}=0, d_{1} \sqrt{\alpha} R^{[1]} e^{-i \theta}-b_{0}-a_{1} \sqrt{\alpha} R e^{-i \theta}=0, \\
& \quad i c_{1} \sqrt{\alpha} R^{[1]} e^{-i \theta}-2 a_{2 x}-i b_{1} \sqrt{\alpha} Q e^{i \theta}=0, i b_{1} \sqrt{\alpha} Q^{[1]} e^{i \theta}-2 d_{2 x}-i c_{1} \sqrt{\alpha} R e^{-i \theta}=0, \\
& \lambda^{1}: i c_{0} \sqrt{\alpha} R^{[1]} e^{-i \theta}-2 a_{1 x}-i b_{0} \sqrt{\alpha} Q e^{i \theta}=0, i d_{0} \sqrt{\alpha} R^{[1]} e^{-i \theta}-2 b_{1 x}-i a_{0} \sqrt{\alpha} R e^{-i \theta}=0, \\
& \quad i a_{0} \sqrt{\alpha} Q^{[1]} e^{i \theta}-2 c_{1 x}-i d_{0} \sqrt{\alpha} Q e^{i \theta}=0, i b_{0} \sqrt{\alpha} Q^{[1]} e^{i \theta}-2 d_{1 x}-i c_{0} \sqrt{\alpha} R e^{-i \theta}=0, \\
& \lambda^{0}: a_{0 x}=b_{0 x}=c_{0 x}=d_{0 x}=0 .
\end{aligned}
$$

The last equation shows $a_{0}, b_{0}, c_{0}, d_{0}$ are functions of $t$ only. Similarly, from

$$
T_{t}+T V=V^{[1]} T,
$$

comparing the coefficients of $\lambda^{j}, j=4,3,2,1,0$, in the same way, we can get

$$
b_{2}=0, c_{2}=0, a_{0 t}=b_{0 t}=c_{0 t}=d_{0 t}=0 .
$$

The last equation shows $a_{0}, b_{0}, c_{0}, d_{0}$ are functions of $x$ only. Therefore, $a_{0}, b_{0}, c_{0}, d_{0}$ are constants.

In order to get the non-trivial solutions, we present a Darboux transformation under the condition $a_{1}=0, d_{1}=0, b_{0}=0, c_{0}=0$. Based on eq.(2.11) and eq.(2.13) and without losing any generality, let Darboux matrix $T$ be in the form of

$$
T_{1}=T_{1}\left(\lambda ; \lambda_{1}, \lambda_{2}\right)=\left(\begin{array}{cc}
a_{2} & 0 \\
0 & d_{2}
\end{array}\right) \lambda^{2}+\left(\begin{array}{cc}
0 & b_{1} \\
c_{1} & 0
\end{array}\right) \lambda+\left(\begin{array}{cc}
a_{0} & 0 \\
0 & d_{0}
\end{array}\right) .
$$

Here $a_{2}, d_{2}, b_{1}, c_{1}$ are undetermined functions of $(x, t)$, which will be expressed by the eigenfunction associated with $\lambda_{1}, \lambda_{2}$ in the Kundu-DNLS spectral problem. First of all, we introduce $n$ eigenfunctions $\psi_{j}$ as

$$
\psi_{j}=\left(\begin{array}{c}
\phi_{j} \\
\varphi_{j}
\end{array}\right), \quad j=1,2, \ldots n, \phi_{j}=\phi_{j}\left(x, t, \lambda_{j}\right), \varphi_{j}=\varphi_{j}\left(x, t, \lambda_{j}\right) .
$$


The elements of one-fold Darboux transformation are parameterized by the eigenfunction $\psi_{1}, \psi_{2}$ associated with $\lambda_{1}, \lambda_{2}$ as

$$
\begin{aligned}
& d_{2}=\frac{1}{a_{2}}, \quad a_{2}=\frac{\varphi_{1} \phi_{2} \lambda_{1}-\phi_{1} \varphi_{2} \lambda_{2}}{\phi_{1} \varphi_{2} \lambda_{1}-\varphi_{1} \phi_{2} \lambda_{2}}, \quad b_{1}=\frac{\phi_{1} \phi_{2}\left(\lambda_{1}^{2}-\lambda_{2}^{2}\right)}{\varphi_{1} \phi_{2} \lambda_{2}-\phi_{1} \varphi_{2} \lambda_{1}}, \\
& c_{1}=\frac{\varphi_{1} \varphi_{2}\left(\lambda_{1}^{2}-\lambda_{2}^{2}\right)}{\phi_{1} \varphi_{2} \lambda_{2}-\varphi_{1} \phi_{2} \lambda_{1}}, \quad a_{0}=d_{0}=\lambda_{1} \lambda_{2} .
\end{aligned}
$$

Note that $\left(a_{2} d_{2}\right)_{x}=0$ is derived from the eq.(2.11), Then we take $a_{2}=\frac{1}{d_{2}}$ in the followings. By transformation eq.(2.11), new solutions are given by

$$
Q^{[1]}=\frac{d_{2}}{a_{2}} Q-\frac{c_{1} e^{-i \theta}}{a_{2} \sqrt{\alpha}}, \quad R^{[1]}=\frac{a_{2}}{d_{2}} R+\frac{b_{1} e^{i \theta}}{d_{2} \sqrt{\alpha}} .
$$

2.2. N-fold Darboux transformation. After considering the One-fold Darboux transformation, let us briefly discuss the N-fold Darboux transformation [33]. First of all, we are in a position to consider the reduction of the Darboux transformation of the Kundu-DNLS equation so that $Q^{[n]}=-\left(R^{[n]}\right)^{*}$. Under this reduction condition, the eigenfunction $\psi_{k}=\left(\begin{array}{c}\phi_{k} \\ \varphi_{k}\end{array}\right)$ associated with eigenvalue $\lambda_{k}$ has following properties [34],

$$
\phi_{2 k+1}^{*}=\varphi_{2 k}, \varphi_{2 k+1}^{*}=\phi_{2 k}, \lambda_{2 k+1}^{*}=\lambda_{2 k}, k=1,2, \ldots n .
$$

Then the Darboux transformation of the DNLS equation is given. Now, the key task is to establish the determinant representation of the n-fold Darboux transformation for KunduDNLS system in this subsection. To this purpose, set

$$
\begin{aligned}
& \mathbf{D}=\left\{\left(\begin{array}{ll}
a & 0 \\
0 & d
\end{array}\right) \mid a, d \text { are complex functions of } x \text { and } t\right\} \\
& \mathbf{A}=\left\{\left(\begin{array}{ll}
0 & b \\
c & 0
\end{array}\right) \mid b, c \text { are complex functions of } x \text { and } t\right\} .
\end{aligned}
$$

According to the form of $T_{1}$ in eq.(2.14), the n-fold Darboux transformation can be represented as

$$
T_{n}=T_{n}\left(\lambda ; \lambda_{1}, \lambda_{2}, \cdots, \lambda_{2 n}\right)=\sum_{i=0}^{2 n} P_{i} \lambda^{i}
$$

with

$$
P_{2 n}=\left(\begin{array}{cc}
a_{2 n} & 0 \\
0 & d_{2 n}
\end{array}\right) \in \mathbf{D}, P_{2 n-1}=\left(\begin{array}{cc}
0 & b_{2 n-1} \\
c_{2 n-1} & 0
\end{array}\right) \in \mathbf{A} .
$$

Here $P_{0}$ is a constant matrix, $P_{i}(1 \leq i \leq 2 n)$ is function of $x$ and $t$.

The n-fold Darboux transformation of the Kundu-DNLS system can be expressed by

$$
T_{n}=T_{n}\left(\lambda ; \lambda_{1}, \lambda_{2}, \cdots, \lambda_{2 n}\right)=\left(\begin{array}{cc}
\frac{\left(T_{n}\right)_{11}}{\Delta_{n}} & \frac{\left(T_{n}\right)_{12}}{\Delta_{n}} \\
\frac{\left(T_{n}\right)_{21}}{\widetilde{\Delta_{n}}} & \frac{\left(T_{n}\right)_{22}}{\widetilde{\Delta_{n}}}
\end{array}\right)
$$


with

$$
\begin{aligned}
& \Delta_{n}=\left|\begin{array}{ccccccc}
\lambda_{1}^{2 n} \phi_{1} & \lambda_{1}^{2 n-1} \varphi_{1} & \lambda_{1}^{2 n-2} \phi_{1} & \lambda_{1}^{2 n-3} \varphi_{1} & \ldots & \lambda_{1}^{2} \phi_{1} & \lambda_{1} \varphi_{1} \\
\lambda_{2}^{2 n} \phi_{2} & \lambda_{2}^{2 n-1} \varphi_{2} & \lambda_{2}^{2 n-2} \phi_{2} & \lambda_{2}^{2 n-3} \varphi_{2} & \ldots & \lambda_{2}^{2} \phi_{2} & \lambda_{2} \varphi_{2} \\
\vdots & \vdots & \vdots & \vdots & \vdots & \vdots & \vdots \\
\lambda_{2 n}^{2 n} \phi_{2 n} & \lambda_{2 n}^{2 n-1} \varphi_{2 n} & \lambda_{2 n}^{2 n-2} \phi_{2 n} & \lambda_{2 n}^{2 n-3} \varphi_{2 n} & \ldots & \lambda_{2 n}^{2} \phi_{2 n} & \lambda_{2 n} \varphi_{2 n}
\end{array}\right| \\
& \left(T_{n}\right)_{11}=\left|\begin{array}{cccccccc}
\lambda^{2 n} & 0 & \lambda^{2 n-2} & 0 & \ldots & \lambda^{2} & 0 & \lambda_{1} \lambda_{2} \ldots \lambda_{2 n} \\
\lambda_{1}^{2 n} \phi_{1} & \lambda_{1}^{2 n-1} \varphi_{1} & \lambda_{1}^{2 n-2} \phi_{1} & \lambda_{1}^{2 n-3} \varphi_{1} & \ldots & \lambda_{1}^{2} \phi_{1} & \lambda_{1} \varphi_{1} & \lambda_{1} \lambda_{2} \ldots \lambda_{2 n} \phi_{1} \\
\lambda_{2}^{2 n} \phi_{2} & \lambda_{2}^{2 n-1} \varphi_{2} & \lambda_{2}^{2 n-2} \phi_{2} & \lambda_{2}^{2 n-3} \varphi_{2} & \ldots & \lambda_{2}^{2} \phi_{2} & \lambda_{2} \varphi_{2} & \lambda_{1} \lambda_{2} \ldots \lambda_{2 n} \phi_{2} \\
\vdots & \vdots & \vdots & \vdots & \vdots & \vdots & \vdots & \vdots \\
\lambda_{2 n}^{2 n} \phi_{2 n} & \lambda_{2 n}^{2 n-1} \varphi_{2 n} & \lambda_{2 n}^{2 n-2} \phi_{2 n} & \lambda_{2 n}^{2 n-3} \varphi_{2 n} & \ldots & \lambda_{2 n}^{2} \phi_{2 n} & \lambda_{2 n} \varphi_{2 n} & \lambda_{1} \lambda_{2} \ldots \lambda_{2 n} \phi_{1}
\end{array}\right|, \\
& \left(T_{n}\right)_{12}=\left|\begin{array}{cccccccc}
0 & \lambda^{2 n-1} & 0 & \lambda^{2 n-3} & \ldots & 0 & \lambda & 0 \\
\lambda_{1}^{2 n} \phi_{1} & \lambda_{1}^{2 n-1} \varphi_{1} & \lambda_{1}^{2 n-2} \phi_{1} & \lambda_{1}^{2 n-3} \varphi_{1} & \ldots & \lambda_{1}^{2} \phi_{1} & \lambda_{1} \varphi_{1} & \lambda_{1} \lambda_{2} \ldots \lambda_{2 n} \phi_{1} \\
\lambda_{2}^{2 n} \phi_{2} & \lambda_{2}^{2 n-1} \varphi_{2} & \lambda_{2}^{2 n-2} \phi_{2} & \lambda_{2}^{2 n-3} \varphi_{2} & \ldots & \lambda_{2}^{2} \phi_{2} & \lambda_{2} \varphi_{2} & \lambda_{1} \lambda_{2} \ldots \lambda_{2 n} \phi_{2} \\
\vdots & \vdots & \vdots & \vdots & \vdots & \vdots & \vdots & \vdots \\
\lambda_{2 n}^{2 n} \phi_{2 n} & \lambda_{2 n}^{2 n-1} \varphi_{2 n} & \lambda_{2 n}^{2 n-2} \phi_{2 n} & \lambda_{2 n}^{2 n-3} \varphi_{2 n} & \ldots & \lambda_{2 n}^{2} \phi_{2 n} & \lambda_{2 n} \varphi_{2 n} & \lambda_{1} \lambda_{2} \ldots \lambda_{2 n} \phi_{1}
\end{array}\right|, \\
& \widetilde{\Delta_{n}}=\left|\begin{array}{ccccccc}
\lambda_{1}^{2 n} \varphi_{1} & \lambda_{1}^{2 n-1} \phi_{1} & \lambda_{1}^{2 n-2} \varphi_{1} & \lambda_{1}^{2 n-3} \phi_{1} & \ldots & \lambda_{1}^{2} \varphi_{1} & \lambda_{1} \phi_{1} \\
\lambda_{2}^{2 n} \varphi_{2} & \lambda_{2}^{2 n-1} \phi_{2} & \lambda_{2}^{2 n-2} \varphi_{2} & \lambda_{2}^{2 n-3} \phi_{2} & \ldots & \lambda_{2}^{2} \varphi_{2} & \lambda_{2} \phi_{2} \\
\vdots & \vdots & \vdots & \vdots & \vdots & \vdots & \vdots \\
\lambda_{2 n}^{2 n} \varphi_{2 n} & \lambda_{2 n}^{2 n-1} \phi_{2 n} & \lambda_{2 n}^{2 n-2} \varphi_{2 n} & \lambda_{2 n}^{2 n-3} \phi_{2 n} & \ldots & \lambda_{2 n}^{2} \varphi_{2 n} & \lambda_{2 n} \phi_{2 n}
\end{array}\right| \\
& \left(T_{n}\right)_{21}=\left|\begin{array}{cccccccc}
0 & \lambda^{2 n-1} & 0 & \lambda^{2 n-3} & \ldots & 0 & \lambda & 0 \\
\lambda_{1}^{2 n} \varphi_{1} & \lambda_{1}^{2 n-1} \phi_{1} & \lambda_{1}^{2 n-2} \varphi_{1} & \lambda_{1}^{2 n-3} \phi_{1} & \ldots & \lambda_{1}^{2} \varphi_{1} & \lambda_{1} \phi_{1} & \lambda_{1} \lambda_{2} \ldots \lambda_{2 n} \varphi_{1} \\
\lambda_{2}^{2 n} \varphi_{2} & \lambda_{2}^{2 n-1} \phi_{2} & \lambda_{2}^{2 n-2} \varphi_{2} & \lambda_{2}^{2 n-3} \phi_{2} & \ldots & \lambda_{2}^{2} \varphi_{2} & \lambda_{2} \phi_{2} & \lambda_{1} \lambda_{2} \ldots \lambda_{2 n} \varphi_{2} \\
\vdots & \vdots & \vdots & \vdots & \vdots & \vdots & \vdots & \vdots \\
\lambda_{2 n}^{2 n} \varphi_{2 n} & \lambda_{2 n}^{2 n-1} \phi_{2 n} & \lambda_{2 n}^{2 n-2} \varphi_{2 n} & \lambda_{2 n}^{2 n-3} \phi_{2 n} & \ldots & \lambda_{2 n}^{2} \varphi_{2 n} & \lambda_{2 n} \phi_{2 n} & \lambda_{1} \lambda_{2} \ldots \lambda_{2 n} \varphi_{1}
\end{array}\right|, \\
& \left(T_{n}\right)_{22}=\left|\begin{array}{cccccccc}
\lambda^{2 n} & 0 & \lambda^{2 n-2} & 0 & \ldots & \lambda^{2} & 0 & \lambda_{1} \lambda_{2} \ldots \lambda_{2 n} \\
\lambda_{1}^{2 n} \varphi_{1} & \lambda_{1}^{2 n-2} \phi_{1} & \lambda_{1}^{2 n-2} \varphi_{1} & \lambda_{1}^{2 n-3} \phi_{1} & \ldots & \lambda_{1}^{2} \varphi_{1} & \lambda_{1} \phi_{1} & \lambda_{1} \lambda_{2} \ldots \lambda_{2 n} \varphi_{1} \\
\lambda_{2}^{2 n} \varphi_{2} & \lambda_{2}^{2 n-1} \phi_{2} & \lambda_{2}^{2 n-2} \varphi_{2} & \lambda_{2}^{2 n-3} \phi_{2} & \ldots & \lambda_{2}^{2} \varphi_{2} & \lambda_{2} \phi_{2} & \lambda_{1} \lambda_{2} \ldots \lambda_{2 n} \varphi_{2} \\
\vdots & \vdots & \vdots & \vdots & \vdots & \vdots & \vdots & \vdots \\
\lambda_{2 n}^{2 n} \varphi_{2 n} & \lambda_{2 n}^{2 n-1} \phi_{2 n} & \lambda_{2 n}^{2 n-2} \varphi_{2 n} & \lambda_{2 n}^{2 n-3} \phi_{2 n} & \ldots & \lambda_{2 n}^{2} \varphi_{2 n} & \lambda_{2 n} \phi_{2 n} & \lambda_{1} \lambda_{2} \ldots \lambda_{2 n} \varphi_{1}
\end{array}\right| .
\end{aligned}
$$

Next, we consider the transformed new solutions $\left(Q^{[n]}, R^{[n]}\right)$ of Kundu-DNLS system corresponding to the n-fold Kundu-DNLS transformation. Under covariant requirement of spectral problem of the Kundu-DNLS system, the transformed form should be

$$
\partial_{x} \psi^{[n]}=\left(J \lambda^{2}+Q^{[n]} \lambda\right) \psi=U^{[n]} \psi
$$

with

$$
\psi=\left(\begin{array}{c}
\phi \\
\varphi
\end{array}\right), \quad J=\left(\begin{array}{cc}
i & 0 \\
0 & -i
\end{array}\right), \quad Q^{[n]}=\left(\begin{array}{cc}
0 & Q^{[n]} \\
R^{[n]} & 0
\end{array}\right)
$$

and then

$$
T_{n x}+T_{n} U=U^{[n]} T_{n}
$$


Substituting $T_{n}$ given by eq.(2.18) into eq.(2.22) and comparing the coefficients of $\lambda^{2 n+1}$, it yields

$$
Q^{[n]}=\frac{d_{n}}{a_{n}} Q-\frac{c_{n-1} e^{-i \theta}}{a_{n} \sqrt{\alpha}}, \quad R^{[n]}=\frac{a_{n}}{d_{n}} R+\frac{b_{n-1} e^{i \theta}}{d_{n} \sqrt{\alpha}} .
$$

Furthermore, taking $a_{n}, d_{n}, b_{n-1}, c_{n-1}$ which are obtained from eq.(2.19) into (2.23), then new solutions $\left(Q^{[n]}, R^{[n]}\right)$ are given by

$$
Q^{[n]}=\frac{\Omega_{21}^{2}}{\Omega_{11}^{2}} Q+\frac{e^{-i \theta}}{\sqrt{\alpha}} \frac{\Omega_{21} \Omega_{22}}{\Omega_{11}^{2}}, \quad R^{[n]}=\frac{\Omega_{11}^{2}}{\Omega_{21}^{2}} R-\frac{e^{i \theta}}{\sqrt{\alpha}} \frac{\Omega_{11} \Omega_{12}}{\Omega_{21}^{2}} .
$$

Here,

$$
\begin{aligned}
& \Omega_{11}=\left|\begin{array}{cccccc}
\lambda_{1}^{2 n-1} \varphi_{1} & \lambda_{1}^{2 n-2} \phi_{1} & \lambda_{1}^{2 n-3} \varphi_{1} & \ldots & \lambda_{1} \varphi_{1} & \phi_{1} \\
\lambda_{2}^{2 n-1} \varphi_{2} & \lambda_{2}^{2 n-2} \phi_{2} & \lambda_{2}^{2 n-3} \varphi_{2} & \ldots & \lambda_{2} \varphi_{2} & \phi_{2} \\
\vdots & \vdots & \vdots & \vdots & \vdots & \vdots \\
\lambda_{2 n}^{2 n-1} \varphi_{2 n} & \lambda_{2 n}^{2 n-2} \phi_{2 n} & \lambda_{2 n}^{2 n-3} \varphi_{2 n} & \ldots & \lambda_{2 n} \varphi_{2 n} & \phi_{2 n}
\end{array}\right| \\
& \Omega_{12}=\left|\begin{array}{cccccc}
\lambda_{1}^{2 n} \phi_{1} & \lambda_{1}^{2 n-2} \phi_{1} & \lambda_{1}^{2 n-3} \varphi_{1} & \ldots & \lambda_{1} \varphi_{1} & \phi_{1} \\
\lambda_{2}^{2 n} \phi_{2} & \lambda_{2}^{2 n-2} \phi_{2} & \lambda_{2}^{2 n-3} \varphi_{2} & \ldots & \lambda_{2} \varphi_{2} & \phi_{2} \\
\vdots & \vdots & \vdots & \vdots & \vdots & \vdots \\
\lambda_{2 n}^{2 n} \phi_{2 n} & \lambda_{2 n}^{2 n-2} \phi_{2 n} & \lambda_{2 n}^{2 n-3} \varphi_{2 n} & \ldots & \lambda_{2 n} \varphi_{2 n} & \phi_{2 n}
\end{array}\right| \\
& \Omega_{21}=\left|\begin{array}{ccccccc}
\lambda_{1}^{2 n-1} \phi_{1} & \lambda_{1}^{2 n-2} \varphi_{1} & \lambda_{1}^{2 n-3} \phi_{1} & \ldots & \lambda_{1} \phi_{1} & \varphi_{1} \\
\lambda_{2}^{2 n-1} \phi_{2} & \lambda_{2}^{2 n-2} \varphi_{2} & \lambda_{2}^{2 n-3} \phi_{2} & \ldots & \lambda_{2} \phi_{2} & \varphi_{2} \\
\vdots & \vdots & \vdots & \vdots & \vdots & \vdots \\
\lambda_{2 n}^{2 n-1} \phi_{2 n} & \lambda_{2 n}^{2 n-2} \varphi_{2 n} & \lambda_{2 n}^{2 n-3} \phi_{2 n} & \ldots & \lambda_{2 n} \phi_{2 n} & \varphi_{2 n}
\end{array}\right| \\
& \Omega_{22}=\left|\begin{array}{|cccccc}
\lambda_{1}^{2 n} \varphi_{1} & \lambda_{1}^{2 n-2} \varphi_{1} & \lambda_{1}^{2 n-3} \phi_{1} & \ldots & \lambda_{1} \phi_{1} & \varphi_{1} \\
\lambda_{1}^{2 n} \varphi_{1} & \lambda_{2}^{2 n-2} \varphi_{2} & \lambda_{2}^{2 n-3} \phi_{2} & \ldots & \lambda_{2} \phi_{2} & \varphi_{2} \\
\vdots & \vdots & \vdots & \vdots & \vdots & \vdots \\
\lambda_{2 n}^{2 n} \varphi_{2 n} & \lambda_{2 n}^{2 n-2} \varphi_{2 n} & \lambda_{2 n}^{2 n-3} \phi_{2 n} & \ldots & \lambda_{2 n} \phi_{2 n} & \varphi_{2 n}
\end{array}\right|
\end{aligned}
$$

So far, we discussed about the determinant construction of n-th Darboux transformation of Kundu-DNLS equation. As an application of these transformations of Kundu-DNLS equation, soliton solutions and positon solutions will be constructed in the next section.

\section{Soliton solutions and Positon solution of the Kundu-DNLS equation}

For $Q=0$ the equations (2.17) is solved by

$$
\psi_{j}=\left(\begin{array}{c}
\phi_{j} \\
\varphi_{j}
\end{array}\right), \quad \phi_{j}=\exp \left(-\frac{i}{8}\left(2 \lambda_{j}{ }^{2} x+\lambda_{j}{ }^{4} t\right)\right), \quad \varphi_{j}=\exp \left(\frac{i}{8}\left(2 \lambda_{j}{ }^{2} x+\lambda_{j}{ }^{4} t\right)\right) .
$$

Considering the choice in eq.(2.17) with $\lambda_{1}=m_{1}+i n_{1}, \lambda_{2}=\lambda_{1}^{*}, m_{1}, n_{1} \in \mathbb{R}$ and using eigenfunctions in eq.(3.29), then one soliton solution of Kundu-DNLS equation is

$$
Q^{[1]}=\frac{\left(e^{i F_{1}} \lambda_{1}-e^{i F_{2}} \lambda_{2}\right)\left(\lambda_{1}+\lambda_{2}\right)}{e^{-2 i f} \sqrt{\alpha}\left(\lambda_{1}-\lambda_{2}\right)},
$$


with $F_{i}=-\frac{1}{4}\left(-2 \lambda_{i}^{2} x+\lambda_{i}^{4} t+4 \theta\right), i=1,2 ; f=\frac{1}{8}\left(\lambda_{1}-\lambda_{2}\right)\left(\lambda_{1}+\lambda_{2}\right)\left(t \lambda_{1}^{2}-2 x+t \lambda_{2}^{2}\right)$. The picture of one soliton solution of the Kundu-DNLS equation and its corresponding density graph are plotted in Fig.1 .
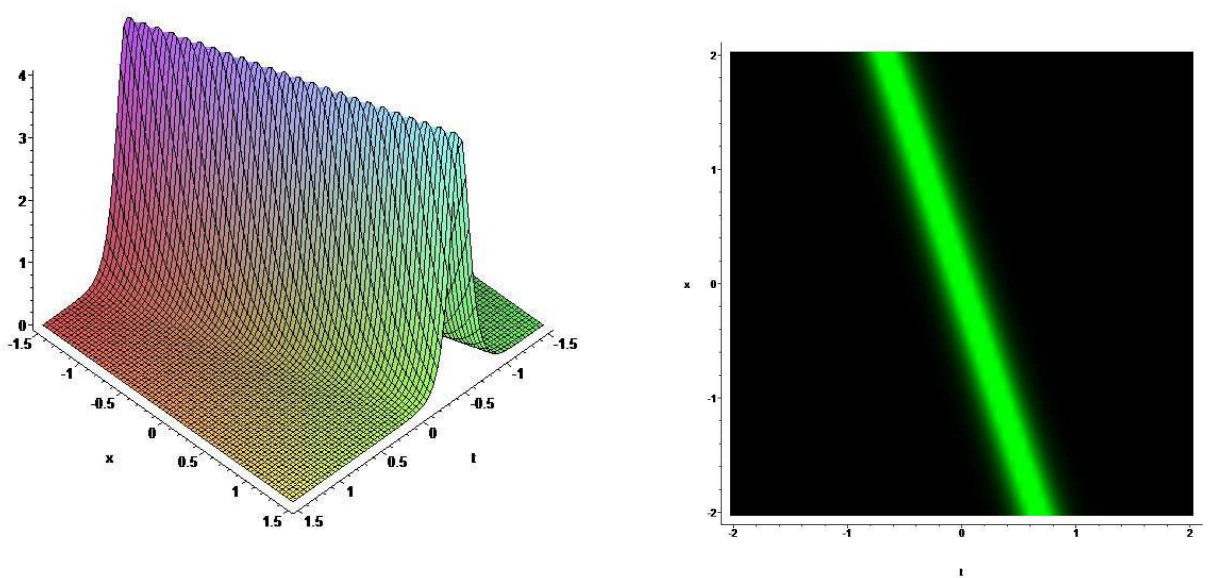

Figure 1. One soliton solution $\left|Q^{[1]}\right|^{2}$ of the Kundu-DNLS equation when $m_{1}=$ $1, n_{1}=2, \alpha=1, \theta=x+t$.

Now let us discuss about the construction of the two-soliton solution of Kundu-DNLS system. For the purpose, we have to use two spectral parameters $\lambda_{1}=m_{1}+i n_{1}, \lambda_{2}=\lambda_{1}^{*}$, and $\lambda_{3}=$ $m_{2}+i n_{2}, \lambda_{4}=\lambda_{3}^{*}$. After two Darboux transformations, the two soliton solution is derived as follows:

$$
Q^{[2]}=\frac{K_{1}}{K_{2}}
$$

where

$$
\begin{aligned}
K_{1}= & -4 i e^{-i \theta}\left(2 M \cos \rho_{1}+H_{1} 0001 e^{\rho_{2}}+H_{2} e^{\rho_{3}}+H_{3} e^{\rho_{4}}+H_{4} e^{\rho_{5}}\right) \\
& \left(H_{5} e^{\rho_{6}}+H_{6} e^{-\rho_{6}^{*}}+H_{7} e^{\rho_{7}}+H_{8} e^{-\rho_{7}^{*}}\right), \\
K_{2}= & -\sqrt{\alpha}\left(2 M \cos \rho_{1}+H_{4} e^{\rho_{2}}+H_{3} e^{\rho_{3}}+H_{2} e^{\rho_{4}}+H_{1} e^{\rho_{5}}\right)^{2}, \\
A= & -\left(m_{1}+m_{2}\right)+i\left(n_{1}+n_{2}\right), \quad B=\left(m_{2}-m_{1}\right)+i\left(n_{1}-n_{2}\right), \\
C= & \left(m_{2}-m_{1}\right)+i\left(n_{1}+n_{2}\right), \quad D=-\left(m_{1}+m_{2}\right)+i\left(n_{1}-n_{2}\right), \\
E= & i n_{1}-m_{1}, \quad F=i n_{2}-m_{2}, \\
H_{1}= & -|A|^{2}|C|^{2} E F, \quad H_{2}=|B|^{2}|D|^{2} E F^{*}, \\
H_{3}= & |B|^{2}|D|^{2} E^{*} F, \quad H_{4}=-|A|^{2}|C|^{2} E^{*} F^{*}, \\
H_{5}= & -m_{1} n_{1} A^{*} B C^{*} D F, \quad H_{6}=m_{1} n_{1} A B^{*} C D F^{*}, \\
H_{7}= & m_{2} n_{2} A^{*} B^{*} C D E, \quad H_{8}=m_{1} n_{1} A B C D^{*} E^{*}, \\
\rho_{1}= & \frac{1}{4}\left(t m_{1}^{4}-t m_{2}^{4}-t n_{2}^{4}+t n_{1}^{4}+6 t m_{2}^{2} n_{2}^{2}-6 t m_{1}^{2} n_{1}^{2}-2 x m_{1}^{2}+2 x m_{2}^{2}+2 x n_{1}^{2}-2 x n_{2}^{2}\right), \\
\rho_{2}= & t m_{1}^{3} n_{1}-t m_{1} n_{1}^{3}+t m_{2}^{3} n_{2}-t m_{2} n_{2}^{3}-x m_{1} n_{1}-x m_{2} n_{2}, \\
\rho_{3}= & t m_{1}^{3} n_{1}-t m_{1} n_{1}^{3}-t m_{2}^{3} n_{2}+t m_{2} n_{2}^{3}-x m_{1} n_{1}+x m_{2} n_{2},
\end{aligned}
$$




$$
\begin{aligned}
& \rho_{4}=-t m_{1}^{3} n_{1}+t m_{1} n_{1}^{3}+t m_{2}^{3} n_{2}-t m_{2} n_{2}^{3}+x m_{1} n_{1}-x m_{2} n_{2}, \\
& \rho_{5}=-t m_{1}^{3} n_{1}+t m_{1} n_{1}^{3}-t m_{2}^{3} n_{2}+t m_{2} n_{2}^{3}+x m_{1} n_{1}+x m_{2} n_{2}, \\
& \rho_{6}=\frac{-i}{4}\left(t m_{1}^{4}+t n_{1}^{4}+4 i t m_{2}^{3} n_{2}-4 i t m_{2} n_{2}^{3}-6 t m_{1}^{2} n_{1}^{2}-2 x m_{1}^{2}+2 x n_{1}^{2}-4 i x m_{2} n_{2}\right), \\
& \rho_{7}=\frac{-i}{4}\left(t m_{2}^{4}+t n_{2}^{4}+4 i t m_{1}^{3} n_{1}-4 i t m_{1} n_{1}^{3}-6 t m_{2}^{2} n_{2}^{2}+2 x m_{2}^{2}+2 x n_{2}^{2}-4 i x m_{1} n_{1}\right) .
\end{aligned}
$$

The picture of two soliton solution of the Kundu-DNLS equation and its corresponding density graph are plotted in Fig.2 .
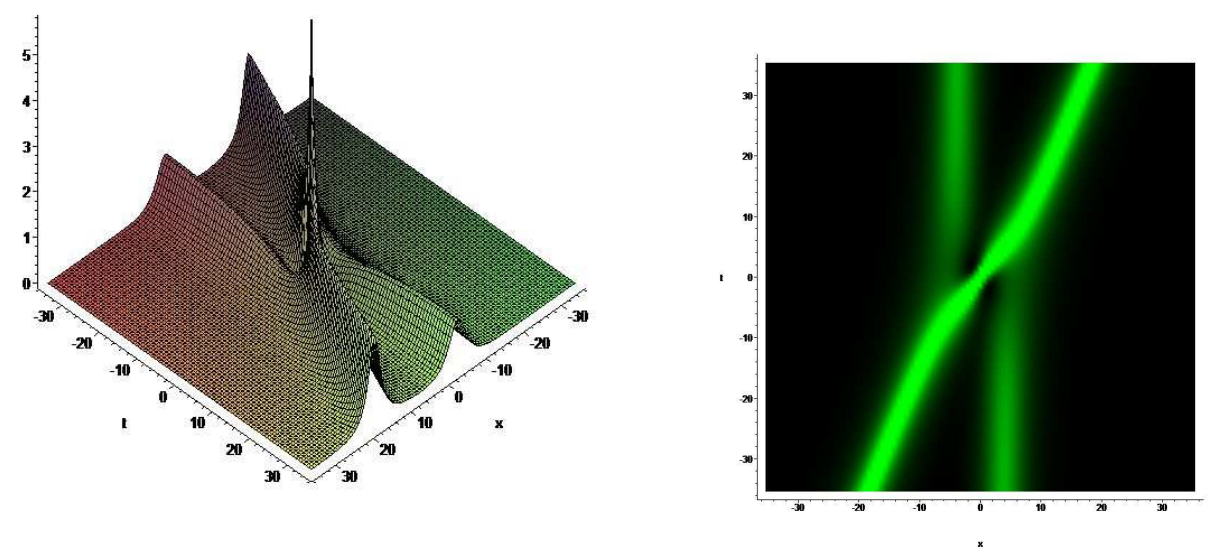

Figure 2. Two soliton solution $\left|Q^{[2]}\right|^{2}$ of the Kundu-DNLS equation when $m_{1}=$ $0.7, m_{2}=0.5, n_{1}=0.3, n_{2}=0.5, \alpha=1$.

In the following, we consider the construction of positon solution of Kundu-DNLS equation in detail. From the two soliton solution, we make use of four spectral parameters $\lambda_{1}=\alpha_{1}+i \beta_{1}$, $\lambda_{2}=\alpha_{1}-i \beta_{1}$ and $\lambda_{3}=\alpha_{1}+i \beta_{1}+\varepsilon, \lambda_{4}=\alpha_{1}-i \beta_{1}+\varepsilon$. By letting the infinitesimal complex number $\varepsilon$ and doing the Taylor expansion of wave function to $\lambda_{1}, \lambda_{2}$, positon solution as the method of ref. [25]. Following four linear functions are used to construct the second positon solution,

$$
\psi_{k}=\left(\begin{array}{c}
\phi_{k} \\
\varphi_{k}
\end{array}\right), \quad \phi_{k}=\exp \left(-\frac{i}{8}\left(2{\lambda_{k}}^{2} x+{\lambda_{k}}^{4} t\right)\right), \quad \varphi_{k}=\exp \left(\frac{i}{8}\left(2 \lambda_{k}{ }^{2} x+\lambda_{k}^{4} t\right)\right)
$$

where $k=1,2,3,4$. The positon solution of the Kundu-DNLS equation is given as follows:

$$
Q_{p}=\frac{-8 \alpha_{1} \beta_{1} G_{1} G_{2}\left(G_{3}+G_{4}\right)}{\left(G_{3}-G_{4}\right)^{2}}
$$

where

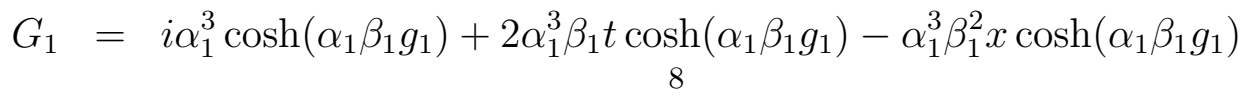




$$
\begin{aligned}
& -\alpha_{1} \beta_{1}^{4} x \cosh \left(\alpha_{1} \beta_{1} g_{1}\right)-\alpha_{1} \beta_{1}^{6} t \cosh \left(\alpha_{1} \beta_{1} g_{1}\right)+3 \alpha_{1}^{5} \beta_{1}^{2} t \cosh \left(\alpha_{1} \beta_{1} g_{1}\right) \\
& -i \alpha_{1}^{6} \beta_{1} t \sinh \left(\alpha_{1} \beta_{1} g_{1}\right)+2 i \alpha_{1}^{4} \beta_{1}^{3} t \sinh \left(\alpha_{1} \beta_{1} g_{1}\right)+i \alpha_{1}^{4} \beta_{1} x \sinh \left(\alpha_{1} \beta_{1} g_{1}\right) \\
& +i \alpha_{1}^{2} \beta_{1}^{3} x \sinh \left(\alpha_{1} \beta_{1} g_{1}\right)+3 i \alpha_{1}^{2} \beta_{1}^{2} t \sinh \left(\alpha_{1} \beta_{1} g_{1}\right)-\beta_{1}^{3} \sinh \left(\alpha_{1} \beta_{1} g_{1}\right), \\
G_{2}= & \cos H_{2}+i \sin H_{2}, \\
G_{3}= & 2 i \alpha_{1}^{3} \beta_{1} \sinh \left(2 \alpha_{1} \beta_{1} g_{1}\right)+4 i \alpha_{1}^{2} \beta_{1}^{6} t-4 i \alpha_{1}^{4} \beta_{1}^{2} x-24 i \alpha_{1}^{4} \beta_{1}^{4} t+ \\
& 2 i \alpha_{1} \beta_{1}^{3} \sinh \left(2 \alpha_{1} \beta_{12000} g_{1}\right)+4 i \alpha_{1}^{6} \beta_{1}^{2} t+4 i \alpha_{1}^{2} \beta_{1}^{2} t+4 i \alpha_{1}^{2} \beta_{1}^{4} x, \\
G_{4}= & \alpha_{1}^{4}+\beta_{1}^{4}-4 \alpha_{1}^{8} \beta_{1}^{2} x t-4 \alpha_{1}^{4} \beta_{1}^{6} x t-4 \alpha_{1}^{6} \beta_{1}^{4} x t+4 \alpha_{1}^{2} \beta_{1}^{8} x t+4 \alpha_{1}^{4} \beta_{1}^{4} x^{2} \\
& +8 \alpha_{1}^{4} \beta_{1}^{8} t^{2}+2 \alpha_{1}^{6} \beta_{1}^{2} x^{2}+2 \alpha_{1}^{10} \beta_{1}^{2} t^{2}+8 \alpha_{1}^{8} \beta_{1}^{4} t^{2}+12 \alpha_{1}^{6} \beta_{1}^{6} t^{2}+2 \alpha_{1}^{2} \beta_{1}^{6} x^{2} \\
& +2 \alpha_{1}^{2} \beta_{1}^{10} t^{2}-\beta_{1}^{4} \cosh \left(2 \alpha_{1} \beta_{1} g_{1}\right)+\alpha_{1}^{4} \cosh \left(2 \alpha_{1} \beta_{1} g_{1}\right), \\
g_{1}= & -x-t \alpha_{1}^{2}-t \beta_{1}^{2}, \\
g_{2}= & x+t+\frac{1}{4} t \beta_{1}^{4}-\frac{3}{2} t \alpha_{1}^{2} \beta_{1}^{2}+\frac{1}{2} x \beta_{1}^{2}+\frac{1}{4} t \alpha_{1}^{4}-\frac{1}{2} x \alpha_{1}^{2} .
\end{aligned}
$$

The picture of positon solution of the Kundu-DNLS equation and its corresponding density graph are plotted in Fig. When $t \rightarrow \infty$, one can find that the difference between the two soliton solution in Fig, 2 and the positon solution in Fig. 3 as following. Positon can be treated as one special case of two solitons. When two spectral parameters of two soliton solution get closer and closer, the separating speed between two branches of two solitons becomes slower and slower. This approximation leads two soliton solutions to one Positon solutions. That is why Positon solutions are also called degenerated solitons. Positons of Kundu-DNLS equation are in fact long-range analogues of solitons of Kundu-DNLS equation and are slowly decreasing, oscillating solutions.
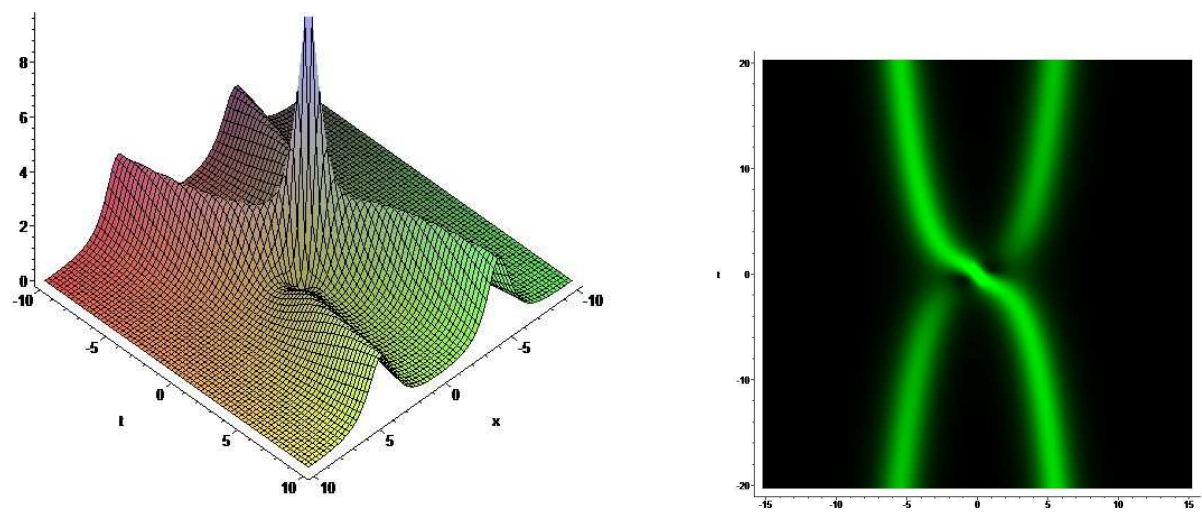

Figure 3. Positon solution $\left|Q_{p}\right|^{2}$ of the Kundu-DNLS equation with $\alpha_{1}=$ $0.8, \beta_{1}=0.8$. 


\section{Breather solution and Rogue wave solutions of the Kundu-DnLS equation}

From last two sections, soliton and positon solutions have been generated for the KunduDNLS equation through the Darboux transformation. However, these solutions were obtained from a trivial seed-zero solution. In this section, we derive the new kind of solution (that is breather solution) from a periodic seed solution.

Taking the seed solution as $Q=c e^{i \rho}, \theta=x+t, \rho=a x+b t$, which admits the constraint in the form

$$
b=-\alpha c^{2} a-2-a^{2}-2 a-\alpha c^{2} .
$$

Then the eigenfunction conrresponded to eigenvalue $\lambda$ is obtained. Using the method of separation of variables and the superposition principle, the eigenfunction $\psi_{k}$ associated with $\lambda_{k}$ is given by

$$
\left(\begin{array}{l}
\phi_{k}\left(x, t, \lambda_{k}\right) \\
\varphi_{k}\left(x, t, \lambda_{k}\right)
\end{array}\right)=\left(\begin{array}{l}
f_{1}\left(x, t, \lambda_{k}\right)[1, k]+f_{2}\left(x, t, \lambda_{k}\right)[1, k]+f_{1}^{*}\left(x, t, \lambda_{k}^{*}\right)[2, k]+f_{2}^{*}\left(x, t, \lambda_{k}^{*}\right)[2, k] \\
f_{1}\left(x, t, \lambda_{k}\right)[2, k]+f_{2}\left(x, t, \lambda_{k}\right)[2, k]+f_{1}^{*}\left(x, t, \lambda_{k}^{*}\right)[1, k]+f_{2}^{*}\left(x, t, \lambda_{k}^{*}\right)[1, k]
\end{array}\right)(
$$

Here

$$
\begin{aligned}
& \left(\begin{array}{l}
f_{1}\left(x, t, \lambda_{k}\right)[1, k] \\
f_{1}\left(x, t, \lambda_{k}\right)[2, k]
\end{array}\right)=\left(\begin{array}{c}
\frac{2-\lambda^{2}+2 a-s}{2 \lambda c} \mathrm{e}^{\frac{1}{8} i\left(-4 a x-4 x-2 x s+t \lambda^{2} s+8 t a+4 t a^{2}+2 t a s+4 t+2 t s+4 t c^{2}+4 t c^{2} a+2 t c^{2} s\right)} \\
\mathrm{e}^{\frac{1}{8} i\left(4 a x+4 x-2 x s+t \lambda^{2} s-8 t a-4 t a^{2}+2 t a s-4 t+2 t s-4 t c^{2}-4 t c^{2} a+2 t c^{2} s\right)}
\end{array}\right), \\
& \left(\begin{array}{l}
f_{2}\left(x, t, \lambda_{k}\right)[1, k] \\
f_{2}\left(x, t, \lambda_{k}\right)[2, k]
\end{array}\right)=\left(\begin{array}{c}
\frac{2-\lambda^{2}+2 a+s}{2 \lambda c} \mathrm{e}^{\frac{-1}{8} i\left(4 a x+4 x-2 x s+t \lambda^{2} s-8 t a-4 t a^{2}+2 t a s-4 t+2 t s-4 t c^{2}-4 t c^{2} a+2 t c^{2} s\right)} \\
\mathrm{e}^{\frac{-1}{8} i\left(-4 a x-4 x-2 x s+t \lambda^{2} s+8 t a+4 t a^{2}+2 t a s+4 t+2 t s+4 t c^{2}+4 t c^{2} a+2 t c^{2} s\right)}
\end{array}\right), \\
& f_{1}\left(x, t, \lambda_{k}\right)=\left(\begin{array}{c}
f_{1}\left(x, t, \lambda_{k}\right)[1, k] \\
f_{1}\left(x, t, \lambda_{k}\right)[2, k]
\end{array}\right), f_{2}\left(x, t, \lambda_{k}\right)=\left(\begin{array}{c}
f_{2}\left(x, t, \lambda_{k}\right)[1, k] \\
f_{2}\left(x, t, \lambda_{k}\right)[2, k]
\end{array}\right), \\
& s=\sqrt{4 a^{2}-4 a \lambda^{2}+8 a+\lambda^{4}-4 \lambda^{2}+4-4 \lambda^{2} c^{2}} .
\end{aligned}
$$

Note that $f_{1}\left(x, t, \lambda_{k}\right)$ and $f_{2}\left(x, t, \lambda_{k}\right)$ are two different solutions of the spectral problem eq.(2.3), but we can only get the trivial solutions through DT of the Kundu-DNLS equation by setting eigenfunction $\psi_{k}$ to be one of them.

Now let us discuss about the construction of the breather solution of Kundu-DNLS equation. For the purpose, we have to use two spectral parameters $\lambda_{1}=\xi+i \eta$, and $\lambda_{2}=\xi-i \eta$. To simplify the calculations, we use the second Darboux transformation discussed in the last section, then the breather solution $Q_{b}$ with $a=-2, c=1, \xi=0.5, \eta=1$ is obtained in the form

$$
Q_{b}=\frac{-b_{1} b_{2}}{2 b_{3}^{2}}
$$

where

$$
\begin{aligned}
b_{1}= & 63508327 i e^{-0.9682458364 i x}+436491673 e^{0.2420614592 t}-563508327 i e^{0.2420614592 t} \\
& -436491673 e^{-0.2420614592 t}-563508327 i e^{-0.2420614592 t}+5 \times 10^{8} i e^{-0.9682458364 i x}, \\
b_{2}= & 1309475019 e^{-0.2420614592 t-2 i x-i t}+10 \times 10^{8} i e^{-0.4 \times 10^{-8} i\left(257938541 x+2.5 \times 10^{8} t\right)} \\
& -1309475019 e^{0.2420614592 t-2 i x-i t}+563508327 i e^{0.2420614592 t-2 i x-i t} \\
& +563508327 e^{-0.2420614592 t-2 i x-i t}+127016654 i e^{-0.4 \times 10^{-8} i\left(742061459 x+2.5 \times 10^{8} t\right)},
\end{aligned}
$$




$$
\begin{aligned}
b_{3}= & 5 \times 10^{8} i e^{-0.9682458364 i x}+63508327 i e^{0.9682458364 i x}-436491673 e^{0.2420614592 t} \\
& -563508327 i e^{0.2420614592 t}+436491673 e^{-0.2420614592 t}-563508327 i e^{-0.2420614592 t} .
\end{aligned}
$$

The picture of breather solutions of the Kundu-DNLS equation and its corresponding density graph are plotted in Fig.4, which propagates along the line $t=0$, however, by changing the value of the parameters, the direction of propagating for the breather will change.
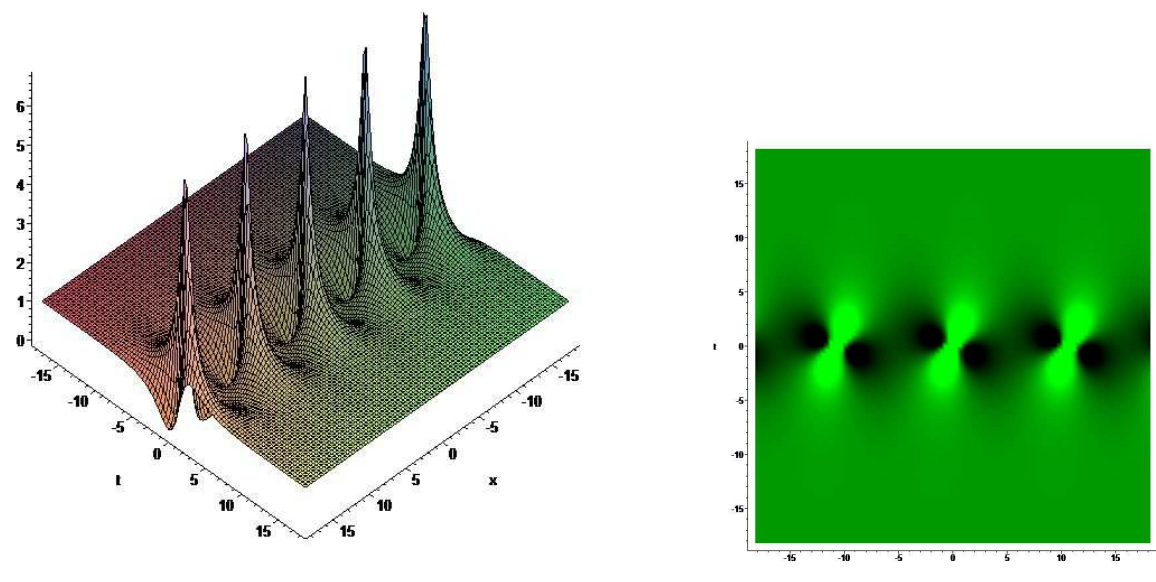

FiguRE 4. Breather solution $\left|Q_{b}\right|^{2}$ of the Kundu-DNLS equation with $a=$ $-2, c=1, \xi=0.5, \eta=1$.

In this section, we construct the rogue wave solution of Kundu-DNLS equation. This kind of solution only appears in some special region of time and space and then drown into a fixed non-vanishing plane. By making use of the Taylor expansion for the breather solution, one order rogue wave solution of $Q_{r}^{[1]}$ for the Kundu-DNLS equation is obtained

$$
Q_{r}^{[1]}=\frac{-v_{1} e^{-i(2 x+t)}}{v_{2}},
$$

where

$$
\begin{aligned}
& v_{1}=3+8 x^{2}+8 i t x^{2}+8 i x t^{2}+8 x t-8 t^{2} x^{2}-4 t^{4}-4 x^{4}+8 i x^{3}-4 i x+12 i t+8 i t^{3}-8 t^{2}, \\
& v_{2}=-1+8 i t^{2}+4 i t+8 i t x^{2}-8 i t^{2} x-8 t x-8 t^{2} x^{2}-8 i x^{3}-4 t^{4}-4 x^{4}-4 i x .
\end{aligned}
$$

The picture of one order rogue wave solution of the Kundu-DNLS equation and its corresponding density graph are plotted in Fig.5. Comparing Fig.1 and Fig.5 can tell us the following important difference between one rogue wave solution and one soliton solution. Rogue wave solution is localized in both $\mathrm{x}$ and $\mathrm{t}$ direction which means it only has large amplitude with a non-vanishing boundary in a small domain of $(\mathrm{x}, \mathrm{t})$ plane. But a soliton solution is a travelling wave and has a vanishing boundary. 

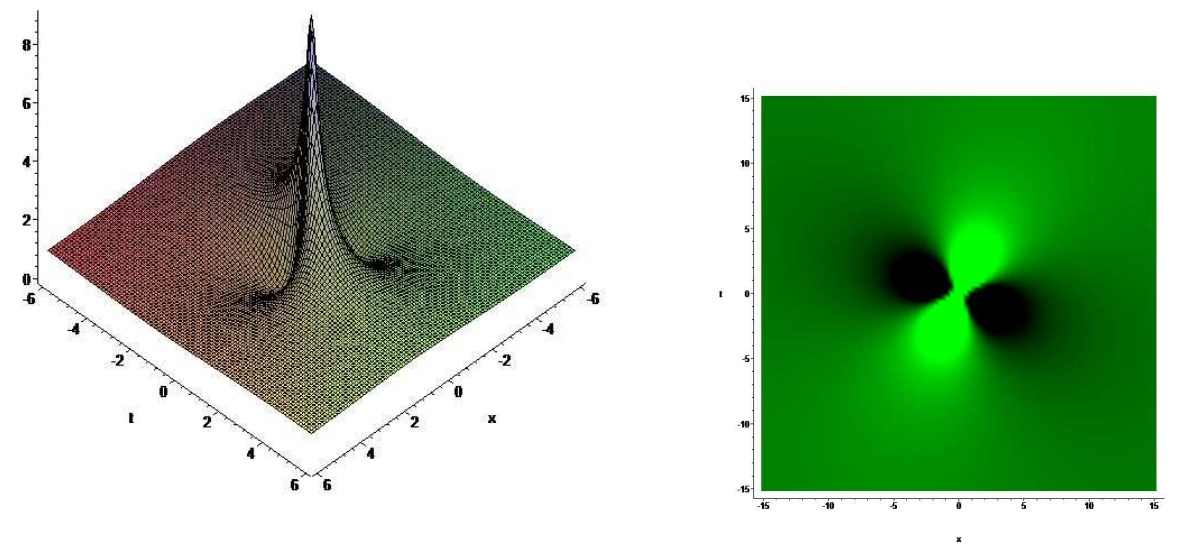

FiguRE 5. One order rogue wave solution $\left|Q_{r}^{[1]}\right|^{2}$ of the Kundu-DNLS equation with $a=-2, c=1, \xi=1, \eta=1$.

When we take $a=-2, c=1, \varepsilon=1, \eta=1$, the specific form of the second rogue wave solution for the Kundu-DNLS equation takes the following form:

$$
Q_{r}^{[2]}=\frac{-v_{3} v_{4} e^{-i(2 x+t)}}{v_{5}^{2}}
$$

where

$$
\begin{aligned}
v_{3}= & -72 x t+48 x^{3} t-216 x^{2} t^{2}+24 x^{2} t^{4}+24 x^{4} t^{2}+90 x^{2}+666 t^{2}-12 x^{4}+180 t^{4}+8 t^{6}+8 x^{6} \\
& +48 x t^{3}+9-48 i x^{3}-48 i x^{3} t^{2}+288 i x t^{2}-54 i x-24 i x t^{4}+24 i t^{5}+24 i x^{4} t+198 i t+336 i t^{3} \\
& +48 i x^{2} t^{3}-24 i x^{5} \\
v_{4}= & 198 x^{2}-45-504 x t+144 x^{3} t+504 x^{2} t^{2}+144 x t^{3}+486 t^{2}+60 t^{4}+60 x^{4}-24 x^{2} t^{4}-8 t^{6} \\
& -24 x^{4} t^{2}-8 x^{6}-48 i x^{3}+24 i x^{5}+48 i x^{3} t^{2}+24 i x t^{4}-288 i x^{4} t-576 i x t^{2}+144 i x^{2} t^{3}-90 i x \\
& -414 i t+72 i x^{4} t+528 i t^{3}+72 i t^{5}, \\
v_{5}= & -48 i x^{3}-48 i x^{3} t^{2}+288 i x t^{2}-54 i x-24 i x t^{4}+72 x t-48 x^{3} t+216 x^{2} t^{2}-24 x^{2} t^{4}-24 i x^{5} \\
& -90 x^{2}-666 t^{2}+24 i t^{5}+12 x^{4}-180 t^{4}-8 t^{6}-8 x^{6}-48 x t^{3}+24 i x^{4} t+198 i t+336 i t^{3}-9 \\
& +48 i x^{2} t^{3}-24 x^{4} t^{2} .
\end{aligned}
$$

The picture of second order rogue wave solution of the Kundu-DNLS equation and its corresponding density graph are plotted in Fig. 6 .

Next, if we replace(4.32) with the following expression,

$$
\left(\begin{array}{c}
\phi_{k}\left(x, t, \lambda_{k}\right) \\
\varphi_{k}\left(x, t, \lambda_{k}\right)
\end{array}\right)=\left(\begin{array}{l}
D_{1} f_{1}\left(x, t, \lambda_{k}\right)[1, k]+D_{2} f_{2}\left(x, t, \lambda_{k}\right)[1, k]+D_{2} f_{1}^{*}\left(x, t, \lambda_{k}^{*}\right)[2, k]+D_{1} f_{2}^{*}\left(x, t, \lambda_{k}^{*}\right)[2, k] \\
D_{1} f_{1}\left(x, t, \lambda_{k}\right)[2, k]+D_{2} f_{2}\left(x, t, \lambda_{k}\right)[2, k]+D_{2} f_{1}^{*}\left(x, t, \lambda_{k}^{*}\right)[1, k]+D_{1} f_{2}^{*}\left(x, t, \lambda_{k}^{*}\right)[1, k]
\end{array}\right)
$$

where 

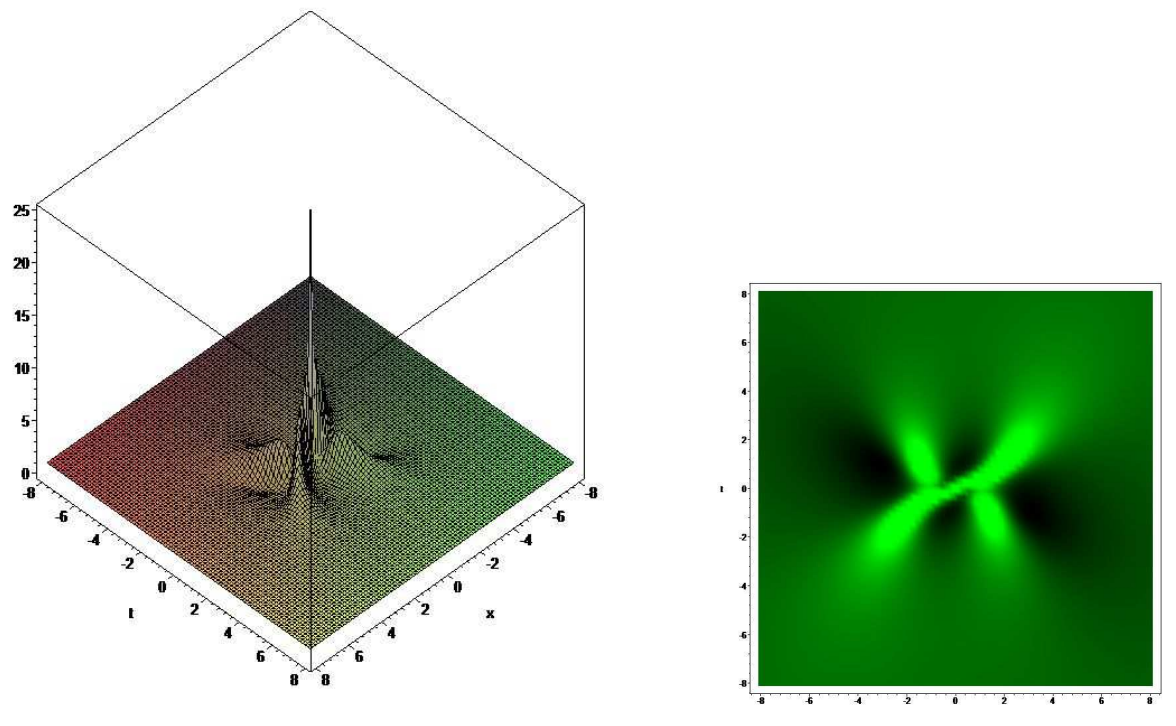

Figure 6 . The second rogue wave solution $\left|Q_{r}^{[2]}\right|^{2}$ of the Kundu-DNLS equation with $a=-2, c=1, \xi=1, \eta=1$.

$$
\left\{\begin{array}{l}
D_{1}=\exp \left(-\mathrm{i} s\left(S_{0}+S_{1} \epsilon+S_{2} \epsilon^{2}\right)\right) \\
D_{2}=\exp \left(\mathrm{i} s\left(S_{0}+S_{1} \epsilon+S_{2} \epsilon^{2}\right)\right)
\end{array}\right.
$$

We can split the second order rogue wave solution into triangle structure with the help of $S_{1}$. A particular structure is displayed in Fig. 7. It is easy to see that three intensity humps appear at different times and space, and each intensity hump is roughly a first-order rogue wave.
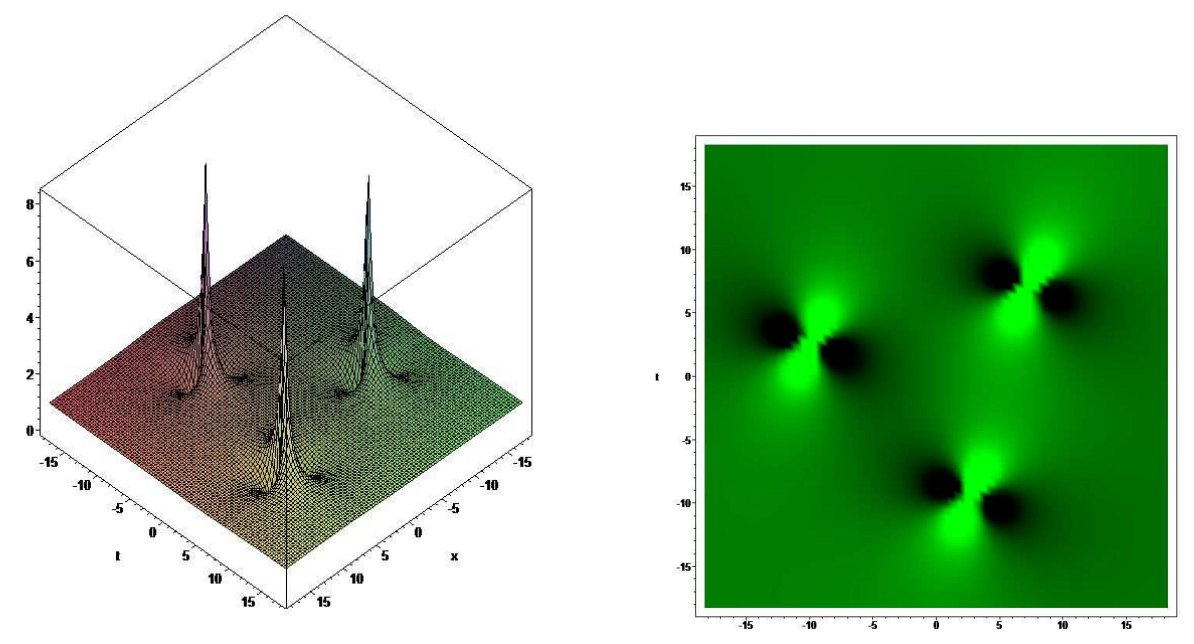

Figure 7. The second rogue wave solution $\left|Q_{r}^{[2]}\right|^{2}$ of the Kundu-DNLS equation with $S_{0}=0, S_{1}=500, S_{2}=0$. 
Next, we examine third-order rogue waves. In this case, Form the figures, We can get thirdorder rogue wave solution with the help of $a=-2, c=1, \alpha=1$. A particular structure is displayed in Fig. 8.
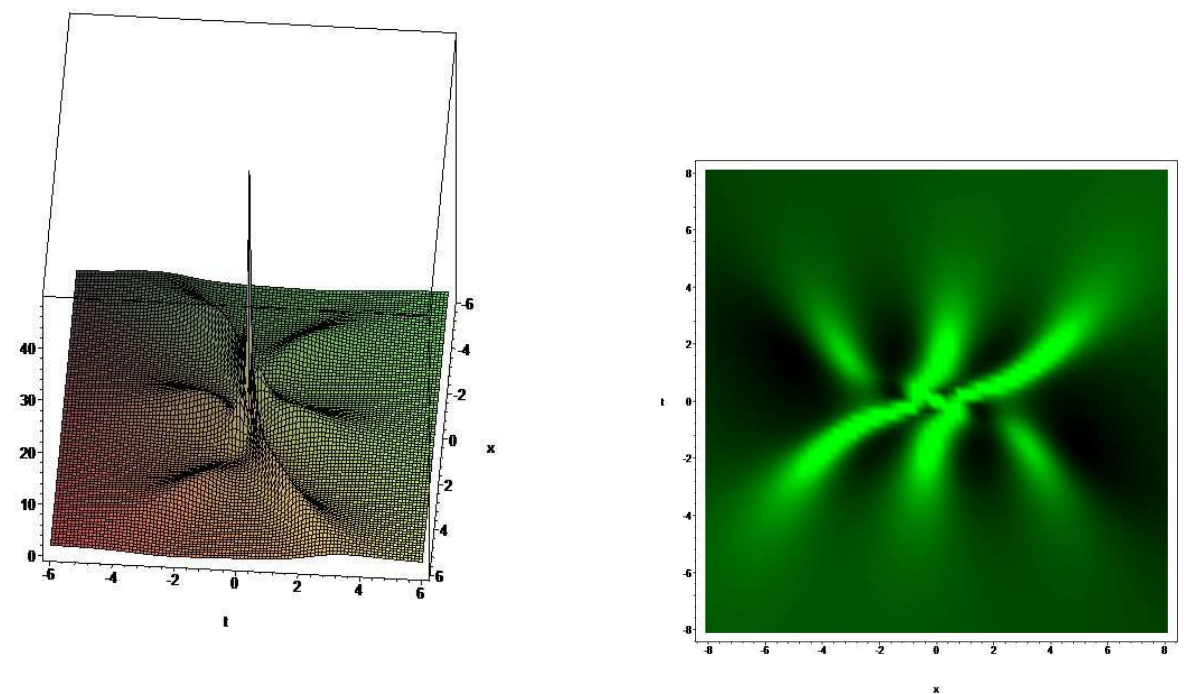

FIGURE 8. The third rogue wave solution $\left|Q_{r}^{[3]}\right|^{2}$ of the Kundu-DNLS equation with $a=-2, c=1, \alpha=1, \varepsilon=0.8, \eta=0.8$.

We can split the third order rogue wave solution into triangle structure with the help of $S_{1}$. A particular structure is displayed in Fig. 9. The third-order rogue wave is seen to possess a regular triangle spatial symmetry structure.
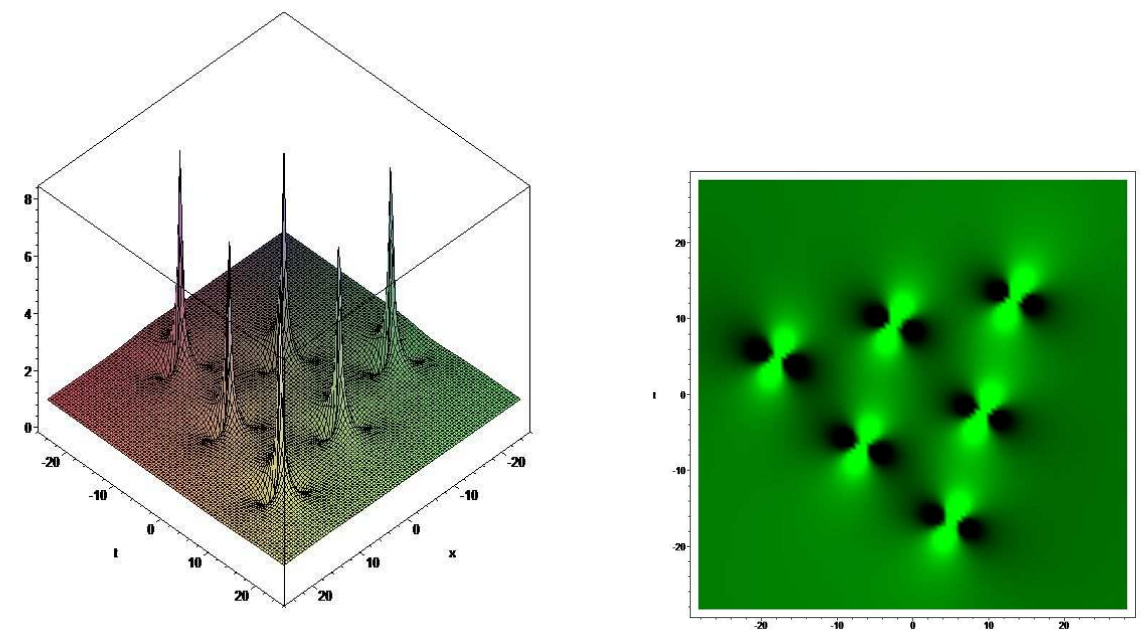

Figure 9. The third rogue wave solution $\left|Q_{r}^{[3]}\right|^{2}$ of the Kundu-DNLS equation with $a=-2, c=1, \alpha=1, S_{0}=0, S_{1}=500, S_{2}=0$.

We can split the third order rogue wave solution into pentagon structure with the help of $S_{2}$. A particular structure is displayed in Fig. 10. The third-order rogue wave exhibits a regular pentagon spatial symmetry structure. 

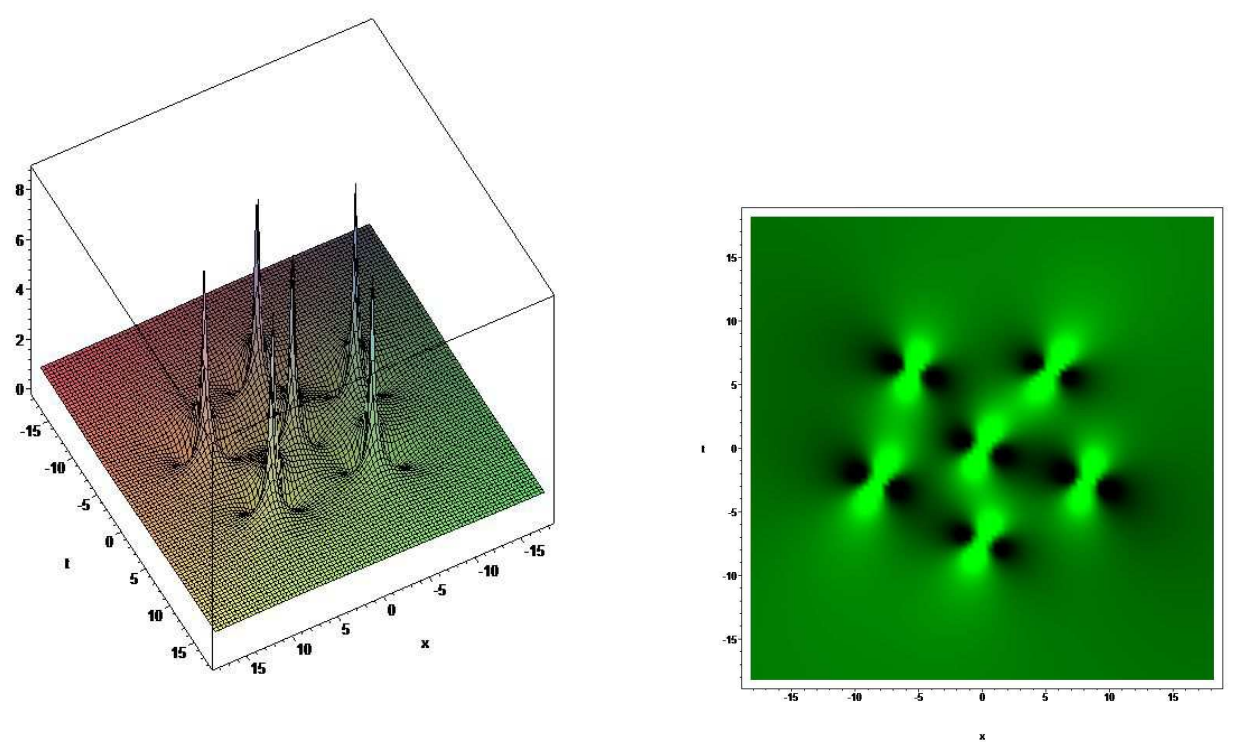

Figure 10. The third rogue wave solution $\left|Q_{r}^{[3]}\right|^{2}$ of the Kundu-DNLS equation with $a=-2, c=1, \alpha=1, S_{0}=0, S_{1}=0, S_{2}=1000$.

From above graphs of rogue wave solutions of the Kundu-DNLS equation, we can find some twisted effect of modified terms of the Kundu-DNLS equation which is different from DNLS equation [2].

\section{CONClusions}

In this paper, we construct the Darboux transformation for the Kundu-DNLS equation. And the determinant representations of the new solution $Q$ of the Kundu-DNLS equation are given. Moreover, by making use of the Darboux transformation, we derive several types of solutions for Kundu-DNLS equation. There solutions include the soliton solutions, positon solution and breather solution, 1-order rogue wave, 2-order rogue wave and 3-order rogue wave. Particularly, these rogue wave solutions possess several free parameters. With the help of these parameters, these rogue waves constitute some patterns, such as fundamental pattern, triangular pattern, circular pattern. On the other hand, we can also derive the higher order rogue wave solutions for Kundu-DNLS equation by making use of the Darboux transformation. The application of high-order rogue in physics will be one interesting subject.

Acknowledgments This work is supported by the NSF of China under Grant No.11271210 and K. C. Wong Magna Fund in Ningbo University. Jingsong He is also supported by Natural Science Foundation of Ningbo under Grant No. 2011A610179. Chuanzhong Li is supported by the National Natural Science Foundation of China under Grant No.11201251, the Natural Science Foundation of Zhejiang Province under Grant No. LY12A01007. We thank Prof. Yishen Li (USTC, Hefei, China) for his long time support and useful suggestions.

\section{REFERENCES}

[1] R. S. Johnson, On the Modulation of Water Waves in the Neighbourhood of $k h \approx 1.363$, Proc. R. Soc. Lond. A, 357, 131-141(1977).

[2] S. W. Xu, J. S. He, L. H. Wang, The Darboux transformation of the derivative nonlinear schrodinger equation, J. Phys. A: Math. Theor., 44, 305203(2011) 
[3] A. Kundu, Integrable Hierarchy of Higher Nonlinear Schrodinger Type Equations, Symmetry, Integrability and Geometry: Methods and Applications, 2, 12(2006).

[4] A. Kundu, Landau-Lifshitz and higher-order nonlinear systems gauge generated from nonlinear schrödinger type equations, J. Math. Phys., 25, 3433(1984).

[5] G. Tanǒglu, Hirota Method for Solving Reaction-Diffusion Equations with Generalized Nonlinearity, International Journal of Nonlinear Science, 1, 30-36(2006).

[6] M. J. Ablowitz and P.A.Clarkson, Solitons, Nonlinear Evolution Equations and Inverse Scattering, J. Fluid Mech, 244, 721-725(1992).

[7] S. Kakei, N. Sasa and J. Satsuma, Bilinearization of a Generalized Derivative Nonlinear schrödinger Equation, Journal of the Physical Society of Japan, 64, 1519-1523(1995).

[8] V. B. Matveev, M. A. Salle,Darboux Transfromations and Solitons, Springer, Berlin(1991).

[9] Y. S. Li, Soliton and integrable system, Shanghai Scientific and Technological Education Press, (1999).

[10] J. S. He, L. Zhang, Y. Cheng and Y. S. Li, Determinant representation of Darboux transformation for the AKNS system, Science in China Series A: Mathematics, 12, 1867-1878(2006).

[11] C. Z. Li, J. S. He, Darboux transformation and positons of the inhomogeneous Hirota and the MaxwellBloch equation, to appear in Science China Physics, Mechanics \& Astronomy, arXiv:1210. 2501.

[12] L. Infeld, T. E. Hull, The Factorization Method, Rev. Mod. Phys., 23, 21-68 (1951).

[13] N. Akhmediev, A. Ankiewicz, and M. Taki,Waves that appear from nowhere and disappear without a trace, Phys. Lett. A, 373, 675-678(2009).

[14] J. L. Tomita, J. L. Jewell, and A. Hasegawa, Generation of subpicosecond solitonlike optical pulses at 0.3 THZ repetion rate by induced modulatinal instability, Appl. Phys. Lett., 49, 236-238(1986).

[15] K. Tai, A. Hasegawa, and A. Tomita, Observation of modulational instability in optical bers, Phys. Rev. Lett, 56, 135-138(1986).

[16] D. R. Solli, C. Ropers, P. Koonath, B. Jalali, Optical rogue waves, Nature, 450, 1054-1057(2007).

[17] K. B. Davis, M. O. Mewes, M. R. Andrews, N. J. van Druten, D. S. Durfee, D. M. Kurn, and W. Ketterle, Bose-Einstein Condensation in a Gas of Sodium Atoms, Phys. Rev. Lett., 75, 3969-3974(1995).

[18] N. N. Akhmediev, V. I. Korneev, and N. V. Mitskevich, N-modulational signals in a single-mode optical fiber with allowance for nonlinearity, Zh. Eksp. Teor. Fiz., 94, 159-170(1988).

[19] N. N. Akhmediev, V. I. Korneev, and N. V. Mitskevich, Modulation instability of a continuous signal in an optical fiber taking into account third-order dispersion, Radiophysics and quantum electronics, 33, 95-100(1990).

[20] J. M. Dudley, G. Genty, F. Dias, B. Kibler, and N. Akhmediev, Modulation instability, Akhmediev Breathers and continuous wave supercontinuum generation, Opt. Express, 17, 21497-21508 (2009).

[21] P. Dubard, P. Gaillard, C. Kleina, and V.B. Matveevb,On multi-rogue wave solutions of the NLS equation and positon solutions of the KdV equation, Eur. Phys. J. Special Topics, 185, 247-258 (2010).

[22] A. Ankiewicz, D. J. Kedziora, N. Akhmediev, Rogue wave triplets, Physics. Letters. A, 375, 2782$2785(2011)$.

[23] D. J. Kedziora, A. Ankiewicz, and N. Akhmediev, Triangular rogue wave cascades, Phys. Rev. E, 86, 056602 (2012).

[24] B. L. Guo, L. M. Ling and Q. P. Liu, Nonlinear Schrodinger equation: Generalized Darboux transformation and rogue wave solutions, Phys. Rev. E, 85, 026607 (2012).

[25] J. S. He, H. R. Zhang, L. H. Wang, K. Porsezian, and A. S. Fokas, A generating mechanism for higher order rogue waves, arXiv: 1209. 3742v3(2012).

[26] C. Z. Li, J. S. He, and K. Porsezian, Rogue waves of the Hirota and the Maxwell-Bloch equation, Physical Review E, 87, 012913(2013).

[27] G. Sanchez-Arriaga, J. R. Sanmartin and S. A. Elaskar, Damping models in the truncated Derivative Nonlinear Schrödinger Equation, Phys. Plasmas, 14, 082108 (2007).

[28] G. Sánchez-Arriaga, T. Hada and Y. Nariyuki, The truncation model of the Derivative Nonlinear Schrödinger Equation, Phys. Plasmas, 16, 042302 (2009).

[29] E. G. Fan, Darboux transformation and Soliton-like solutions for the Gerdjikov-Ivanov equation, J. Phys. A: Math. Gen., 33, 6925-6933(2000).

[30] D. J. Kaup and A. C. Newell, An exact solution for a Derivative Nonlinear Schrödinger equation, J. Math. Phys., 19, 798-801(1978). 
[31] J. C. He, Z. Y. Chen, Comment on Revision of Kaup Newell's Works on IST for DNLS Equation, Commun. Theor. Phys., 50, 1369-1374(2008).

[32] T. Kawata, N. Kobayashi and H. Inoue, Soliton solution of the Derivative Nolinear Schrödinger equation, J. Phys. Soc. Japan, 46, 1008-1015(1979).

[33] G. Q. Zhou and N. N. Huang, An N-soliton solution to the DNLS equation based on revised inverse scattering transform, J. Phys. A: Math. Theor., 40, 13607-13623(2007).

[34] K. Imai, Generlization of Kaup-Newell Inverse Scattering Formulation and Darboux Transformation, J. Phys. Soc. Japan, 68, 355-359(1999). 